\title{
Improved reproducibility in genome-wide DNA methylation analysis for PAXgene-fixed samples compared with restored formalin-fixed and paraffin-embedded DNA
}

\author{
Gitte Brinch Andersen $^{\mathrm{a}, \mathrm{b}}$, Henrik Hager ${ }^{\mathrm{c}}$, Lise Lotte Hansen ${ }^{\mathrm{a}}$, Jörg Tost ${ }^{\mathrm{b}, *}$ \\ ${ }^{a}$ Department of Biomedicine, Aarhus University, DK-8000 Aarhus C, Denmark \\ ${ }^{\mathrm{b}}$ Laboratory for Epigenetics and Environment, Centre National de Génotypage, CEA-Institut de Génomique, 91000 Evry, France \\ ${ }^{\mathrm{c}}$ Department of Pathology, Aarhus University Hospital, DK-8000 Aarhus C, Denmark
}

\section{A R T I C L E I N F O}

\section{Article history:}

Received 27 May 2014

Received in revised form 28 August 2014

Accepted 9 September 2014

Available online 30 September 2014

\section{Keywords:}

PAXgene

DNA methylation

Illumina 450K BeadChip

FFPE

DNA restoration

Genome-wide methylation analysis

\begin{abstract}
A B S T R A C T
Formalin fixation has been the standard method for conservation of clinical specimens for decades. However, a major drawback is the high degradation of nucleic acids, which complicates its use in genome-wide analyses. Unbiased identification of biomarkers, however, requires genome-wide studies, precluding the use of the valuable archives of specimens with long-term follow-up data. Therefore, restoration protocols for DNA from formalin-fixed and paraffin-embedded (FFPE) samples have been developed, although they are cost-intensive and time-consuming. An alternative to FFPE and snapfreezing is the PAXgene Tissue System, developed for simultaneous preservation of morphology, proteins, and nucleic acids. In the current study, we compared the performance of DNA from either PAXgene or formalin-fixed tissues to snap-frozen material for genome-wide DNA methylation analysis using the Illumina 450K BeadChip. Quantitative DNA methylation analysis demonstrated that the methylation profile in PAXgene-fixed tissues showed, in comparison with restored FFPE samples, a higher concordance with the profile detected in frozen samples. We demonstrate, for the first time, that DNA from PAXgene conserved tissue performs better compared with restored FFPE DNA in genome-wide DNA methylation analysis. In addition, DNA from PAXgene tissue can be directly used on the array without prior restoration, rendering the analytical process significantly more time- and cost-effective.
\end{abstract}

(c) 2014 Elsevier Inc. All rights reserved.
Molecular diagnostics and clinical research are largely dependent on a broad spectrum of biomolecules for accurate diagnosis, prediction of outcome, and selection of appropriate therapies using well-annotated samples with long-term follow-up data. During recent years, personalized medicine has become a focus of research, and the search for novel and better performing biomarkers has intensified. The quality of the biomarker, and thereby the outcome of the analytical results, is dependent on many preanalytical factors. Sample storage is one of the most important factors, and the lack of highly preserved tissue samples has been pointed out as one of the major drawbacks to identifying new biomarkers [1]. Formalin fixation and paraffin-embedding (FFPE) ${ }^{1}$ has been the standard treatment for biopsies and surgical specimens for many

\footnotetext{
* Corresponding author. Fax: +33 (0)1 60878485.

E-mail address: tost@cng.fr (J. Tost).

1 Abbreviations used: FFPE sample, formalin-fixed and paraffin-embedded sample; $\mathrm{CpG}$, cytosine followed by guanine; PFPE, PAXgene-fixed paraffin-embedded; $C I$, confidence interval.
}

decades [2]. As a result, most pathology departments have collected large archives of FFPE samples together with long-term clinical follow-up, making these collections an extremely valuable resource for biomedical research. The main drawback of formalin fixation, however, is the crosslinking between DNA and proteins caused by the formalin [3]. The harsh conditions required to break this crosslinking limit the use of the biomolecules isolated from FFPE tissue in molecular analysis $[4,5]$. Therefore, the quality and yield of DNA is generally far lower compared with DNA obtained from fresh frozen tissue [6,7]. Snap-freezing of tissues in liquid nitrogen is widely accepted as the "gold standard" for the preservation of proteins and nucleic acids. However, this method often causes morphological changes in the tissue due to intracellular ice formation [8], compromising the histology of the tissue [9].

An alternative to FFPE and fresh frozen preservation is the noncrosslinking PAXgene Tissue System (PreAnalytiX, Hombrechtikon, Switzerland), which has proven to be efficient for preservation of the morphology and allows high-quality extraction of proteins, RNA, and DNA from the fixed tissues [10-13]. 
The focus on high-quality molecules has increased as the search for new biomarkers has intensified, especially in cancer research. It is now well accepted that the required molecular alterations for neoplastic initiation and progression can, in addition to genetic changes, be acquired through epigenetic mechanisms [14]. These are mitotically heritable changes in gene expression that are not caused by underlying changes in the primary DNA sequence, comprising DNA methylation, histone modifications, and changes in the expression patterns of noncoding RNAs. DNA methylation is the most widely studied epigenetic mechanism and occurs predominantly at cytosines followed by guanine (CpGs). Acquisition of DNA methylation at normally unmethylated $\mathrm{CpG}$ islands in the promoter regions of genes is often correlated with transcriptional silencing of the associated genes [15-17]. Changes of DNA methylation levels and patterns have been widely studied in cancer research, where interest has focused on biomarkers for predicting cancer development, diagnosis, prognostics, and response to treatment $[18,19]$ but have recently been shown to also be of great importance in many complex diseases $[20,21]$.

The Infinium 450K Human Methylation BeadChip (Illumina, San Diego, CA, USA) is an array-based readout investigating more than $450,000 \mathrm{CpG}$ sites. It has become a method of choice for genomewide DNA methylation analysis because it allows, for the first time, analyzing a relatively high number of $\mathrm{CpGs}$ throughout the human genome at a reasonable price [22]. However, DNA extracted from FFPE samples is not suitable for direct use in genome-wide epigenotyping studies because the DNA is inefficiently amplified due to the high degradation. A restoration method for FFPE DNA, developed by Thirlwell and coworkers [23], uses a ligation-based approach to obtain DNA fragments of sufficient size. However, there is still a controversy about the concordance between differentially methylated loci detected in fresh frozen tissue and restored DNA from FFPE tissue [24]. A commercial FFPE restoration kit (Illumina) has been developed for the 450K Human Methylation array, which performs quality control of the DNA to assess its usability for the 450K array and restores the DNA to an acceptable quality using a similar ligation-based DNA repair approach.

In the current study, we investigated the performance of DNA purified from PAXgene-fixed tissue for genome-wide DNA methylation analysis and compared these results with restored DNA from FFPE tissue from the same tumor using the Illumina restoration kit. Fresh frozen tissue from the same samples was used as reference.

\section{Materials and methods}

\section{Samples}

Matched fresh frozen, FFPE, and PAXgene-fixed paraffin-embedded (PFPE) blocks from surgical specimens from 10 patients diagnosed with lung adenocarcinoma and 4 paired normal lung samples were collected from the archives of the Institute of Pathology at Aarhus University Hospital (Denmark). The specimens were 1 to 2 years old. The ethical committee of the Central Denmark Region approved this study.

\section{DNA extraction from fresh frozen tissue}

The tumors were cut into small pieces immediately after surgery, snap-frozen, and stored at $-80^{\circ} \mathrm{C}$ until further use. For each sample, 10 tissue sections of $10 \mu \mathrm{m}$ were used for DNA extraction. The cryostat-cut frozen sections were incubated overnight at $55^{\circ} \mathrm{C}$ with $150 \mu \mathrm{l}$ of proteinase $\mathrm{K}(10 \mathrm{mg} / \mathrm{ml})$ in a thermomixer (1000 rpm). DNA extraction was subsequently performed using the ChargeSwitch gDNA Micro Tissue Kit (Life Technologies, St. Aubin, France) according to the manufacturer's instructions.

\section{DNA extraction from FFPE and PAXgene preserved tissue}

For each sample, 3 tissue sections of $10 \mu \mathrm{m}$ from either the FFPE or PAXgene preserved tissue were used for DNA extraction. DNA was extracted using the QIAamp DNA FFPE kit according to the manufacturer's instructions. For the extraction of PAXgene preserved samples, a slight modification to the protocol was made: after addition of the ATL buffer provided in the QIAamp DNA FFPE kit and incubation at $56{ }^{\circ} \mathrm{C}$, the sample was not incubated at $90^{\circ} \mathrm{C}$ for $1 \mathrm{~h}$ because the tissue was not formalin fixed and, therefore, no methylene bridges needed to be broken.

\section{DNA quantitation}

DNA quantitation was measured using the Quant-iT dsDNA Broad-Range Assay Kit (Life Technologies) following the manufacturer's instructions. The FFPE tumor sample 4 did not contain enough DNA after purification and was omitted from further processing.

\section{FFPE DNA restoration}

DNA extracted from FFPE tissue needed to be restored before analysis on the Infinium 450K Methylation BeadChip. The quality of the FFPE DNA was verified with the Illumina FFPE quality control kit according to the manufacturer's instructions with a slight modification in the qPCR program, that is, $10 \mathrm{~s}$ instead of $30 \mathrm{~s}$ at $95{ }^{\circ} \mathrm{C}$ in the 40 cycles. The quality control kit verified the presence of amplifiable DNA in the FFPE samples. All samples passed the quality control. After bisulfite treatment (see below), sample volume was reduced from 70 to $16 \mu \mathrm{l}$ at $65{ }^{\circ} \mathrm{C}$ to obtain a DNA quantity of $250 \mathrm{ng}$ in a final volume of $8 \mu \mathrm{l}$, which is required for the FFPE restoration. FFPE DNA was restored using the Infinium HD FFPE Restore Kit (Illumina) according to the manufacturer's instructions.

\section{DNA methylation profiling}

The Infinium 450K Methylation BeadChip was used for genomewide methylation analysis. The experimental protocol was followed according to the manufacturer's instructions using $200 \mathrm{ng}$ of DNA of each sample, which was bisulfite-converted using the EpiTect Bisulfite Conversion Kit (Qiagen, Hilden, Germany). The DNA extracted from the PAXgene preserved tissue was directly used on the array, whereas DNA from the FFPE tissue needed to be restored prior to hybridization, as described above. BeadChips were scanned using the Illumina iScan system. The methylation level of each CpG site was calculated as the methylation $\beta$ value using the intensities between methylated and unmethylated probes $(\beta$ value $=$ methylated probe intensity $(\mathrm{M}) /[$ unmethylated probe intensity $(U)+$ methylated probe intensity $(M)+100])$ as defined by Illumina. Data were extracted using Genome Studio software version 2011.1, Methylation module version 1.9.0 (Illumina), without any normalization steps. The FFPE tumor 1 duplicate 2 sample was excluded from further analysis because this sample failed on the Infinium $450 \mathrm{~K}$ array, with only $40 \%$ of the probes having a detection $P$ value less than 0.01 . The probe intensities were in the same range for all three fixation methods. There was no correlation between the length of formalin fixation and the quality of the data as assessed by the number of valid $P$ values or the coefficient of determination $\left(R^{2}\right)$ in the linear correlation analysis.

\section{Data analysis}

Mean methylation difference, 95\% limit of agreement, and 95\% confidence interval $(C I)$ were calculated using Stata Statistical Software, release 13 [25]. 
$\mathrm{R}$ version 3.0.0 [26] was used for the Pearson correlation analysis, McNemar's test, density plots, and Bland-Altman plots with in-house developed scripts. Bland-Altman plots have been developed for the comparison of two methods and yield the interval in which $95 \%$ of future observations will fall [27]. The mean difference indicates whether a fixed bias exists, and the limit of agreement shows how far the measurements for the two methods are apart. The smaller the intervals of the limit of agreement are, the closer the resemblance between the two methods is. The Bland-Altman limit of agreement is based on a $t$ distribution, which makes it possible to calculate a $C I$ value for the lower and higher limits of agreement [28]. Unsupervised hierarchical clustering was performed using $\beta$ values for distance calculation and the Ward method for distance measure.

Non-normalized $\beta$ values were used to identify differentially methylated probes between tumor and normal tissues. Probes with a detection $P$ value greater than 0.01 were removed from the analysis. The genes were retrieved separately for each fixation group. Three different probe lists were obtained for each group with a cutoff of 20,25 , or $30 \%$ methylation difference and a Mann-Whitney test with a $P$ value less than 0.05 between normal lung and tumor tissues. Venn analysis was performed in BioVenn. McNemar's test was used to calculate the $P$ value for the difference between FFPE and PAXgene resemblance with fresh frozen genes.

\section{Results}

The aim of the current study was to evaluate whether DNA extracted from PAXgene-fixed specimens may present a useful resource for genome-wide DNA methylation analysis using the Illumina Infinium 450K BeadChip. To investigate the performance of DNA from PAXgene preserved tissue and DNA obtained after restoration from FFPE tissue, several analyses were carried out to compare the results with DNA methylation profiles obtained from matched fresh frozen samples.

\section{Correlation between methods for sample preservation}

An unsupervised hierarchical cluster analysis was performed to investigate the similarity of the $\beta$ values for each fixation method (Fig. 1). As expected, most lung tumor samples clustered together in one cluster, and all normal lung samples clustered in the second main cluster also containing 3 tumor samples. However, for tumor sample 5, it was only the FFPE sample that clustered with the normal samples. Seven of the samples showed a closer resemblance between PAXgene and fresh frozen tissue. Only 2 samples had a closer similarity between FFPE and fresh frozen tissue. However, for these the PAXgene samples were still close to the fresh frozen samples, whereas the corresponding FFPE samples clustered farther away when PAXgene and fresh frozen samples clustered together. Four samples had PAXgene and FFPE as the most alike samples. A multidimensional scaling plot confirmed the results from the cluster analysis with clustering by sample and not by preservation method (data not shown).

To assess the similarity between the DNA methylation profiles obtained from PAXgene preserved tissues, a linear correlation analysis was performed using the $\beta$ values of matching PAXgene and fresh frozen samples. The coefficient of determination $\left(R^{2}\right)$ between the PAXgene and corresponding frozen samples was between 0.9200 and 0.9904 for the 10 tumor and 4 normal samples (Fig. 2; see also Fig. S1 in online Supplementary material), respectively. These results indicate high consistency between the measured $\beta$ values of PAXgene preserved and fresh frozen tissues, suggesting that PAXgene preserved tissue had DNA quality close to that of the fresh frozen samples. The reproducibility of the DNA methylation patterns from PAXgene and fresh frozen DNA was investigated for 2 samples analyzed in duplicates. Both PAXgene and fresh frozen tissues showed good reproducibility (see Fig. S2 in Supplementary material), supporting the observation that the quality of PAXgene preserved DNA is comparable to that of DNA from fresh frozen tissue.

The observed correlation between restored FFPE and fresh frozen samples was between 0.8545 and 0.9873 for 9 tumor and 4 normal samples (Fig. 3; see also Fig. S3), respectively, and thus was lower compared with the corresponding PAXgene samples. The FFPE sample corresponding to tumor sample 1 duplicate 2 failed the experimental procedure, and generally more probes failed in the FFPE samples compared with the fresh frozen or PAXgene samples (detection $P$ value $>0.01$ ). On average, 6422 (1.32\%; $C I[1.29,1.36])$ probes failed and were removed per FFPE sample, whereas only $541(0.11 \%$; $C I[0.10,0.12])$ probes per fresh frozen sample and 363 (0.08\%; CI [0.067, 0.083]) probes per PAXgene sample showed insufficient confidence in the measured $\beta$ value. This again indicated a higher quality for DNA from PAXgene tissue compared with restored FFPE DNA. However, it should be noted that, in general, $\beta$ values of restored FFPE DNA also correlated well with those obtained from fresh frozen DNA, although to a lesser extent.

The correlation analyses should, however, be viewed with caution because a high correlation coefficient does not necessarily indicate a resemblance between the two tissue specimens. The correlation coefficient only describes how well the regression line fits each measure point and does not necessarily describe how similar the data from the two preservation methods are.

\section{Distribution of mean methylation differences}

The similarity between the DNA methylation profile measured from PAXgene or restored FFPE preserved tissue compared with the one measured from fresh frozen tissue was analyzed using the mean methylation difference $(\Delta \beta$-PAXgene $=\beta$ value PAXgene $-\beta$ value fresh frozen; $\Delta \beta$-FFPE restored $=\beta$ value FFPE restored $-\beta$ value fresh frozen). The distribution of the mean methylation difference for both tumor and normal DNA from PAXgene and restored FFPE tissues compared with the respective fresh frozen samples was centered close to zero (Fig. 4). Nine PAXgene samples had a mean methylation difference closer to zero than the corresponding FFPE samples, and only 5 FFPE samples had a mean methylation difference closer to zero than the corresponding PAXgene samples (Table 1). The lung tumor FFPE samples deviated more from the fresh frozen samples than the normal lung FFPE samples. This may be a result of the unstable environment caused by necrosis and apoptosis in the tumor samples, making the DNA more vulnerable to degradation caused by the formalin fixation. It may also be explained by tumor heterogeneity given that the sections from the FFPE samples and the fresh frozen samples are not from the exact same location of the tumors.

This analysis investigated a difference in the global mean methylation difference of all probes in the paired samples. It did not permit assessing the differences between the paired measures for each individual probe.

To determine how similar the two methods were, the limit of agreement for the difference between the two methods was calculated based on Bland-Altman plots (Table 2; see also Fig. S4). Both PAXgene and FFPE methylation values deviated from the values measured on fresh frozen tissue. For 8 samples, PAXgene had a smaller limit of agreement than the corresponding FFPE sample, whereas 4 samples had almost the same range of the limit of agreement for both PAXgene and FFPE samples and only 1 sample had a smaller limit of agreement in the FFPE tissue compared with the corresponding PAXgene tissue. This indicated that the methylation level for each probe in the PAXgene preserved tissue more closely 


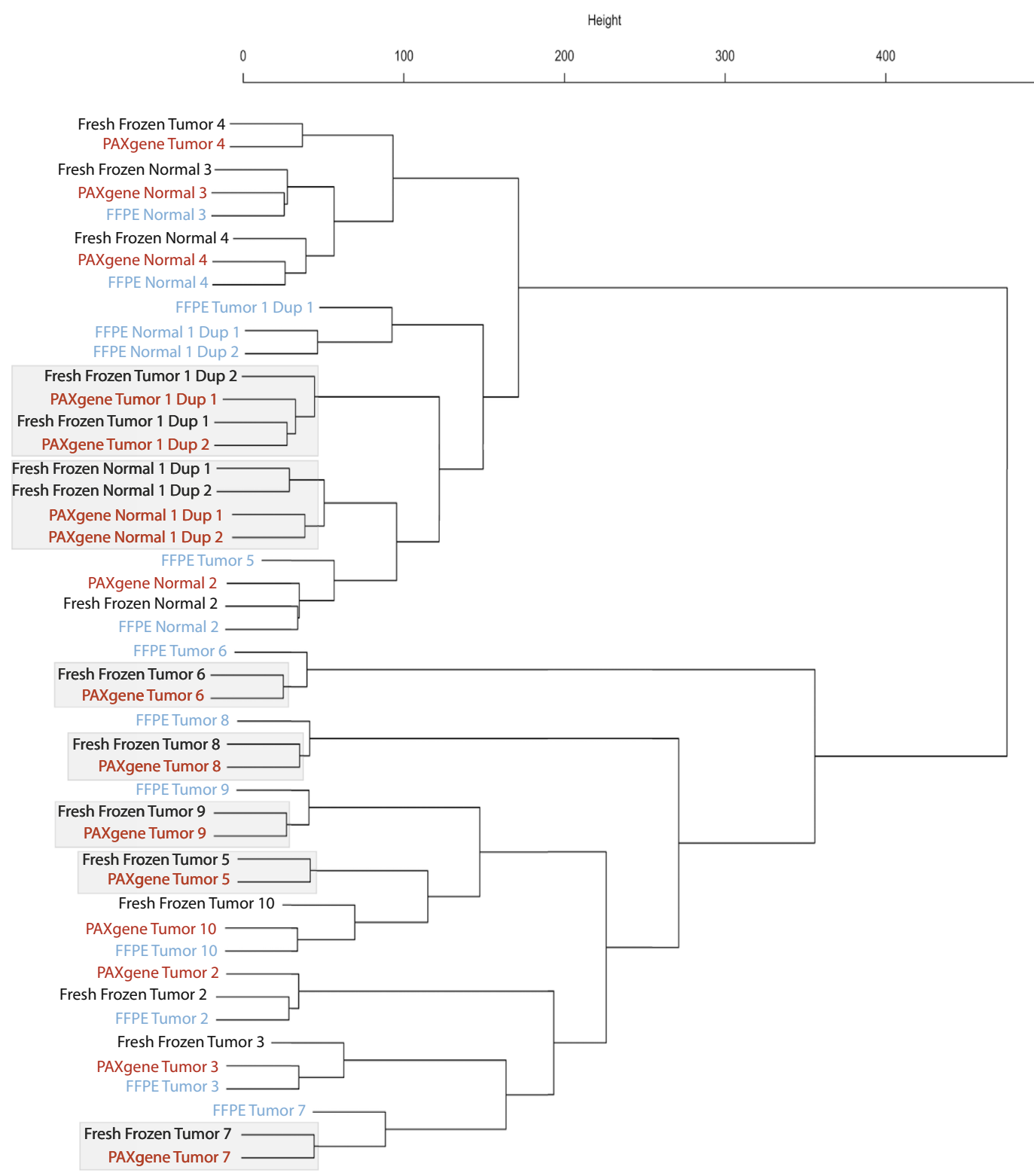

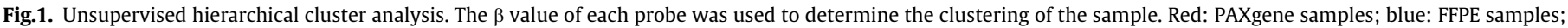

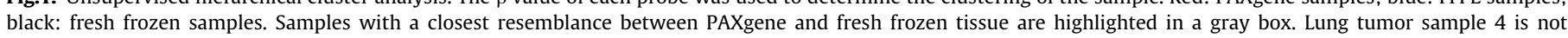
highlighted because the FFPE sample was not investigated for DNA methylation due to a low amount of DNA.

resembled the methylation measured in the fresh frozen DNA than the restored FFPE DNA did. The above-described cluster analysis was also in good agreement with the limit of agreement analysis. This analysis further supported a closer resemblance of DNA methylation measures between PAXgene and fresh frozen DNA compared with restored FFPE DNA and fresh frozen DNA.

\section{Detection of differentially methylated probes}

The resemblance of the profile of differentially methylated probes was assessed through separate differential DNA methylation analysis between tumor and normal lung tissues for each fixation method (Fig. 5). A conservative methylation difference of more than $30 \%$ between tumor and normal tissues and a $P$ value less than 0.05 was set as the threshold. The largest number of probes was identified in the restored FFPE tissue, and the fewest number of probes was identified in the PAXgene tissue. However, when using the probe list obtained from the fresh frozen tissue as a reference, the probes identified in PAXgene-fixed samples were $54.3 \%$ ( $C I[52.1,56.5])$ in agreement with the fresh frozen DNA, whereas FPPE concurred in only 30.0\% (CI [28.0, 32.0]) with the fresh frozen samples. The difference between probes identified in the FFPE or PAXgene and fresh frozen samples was statistically significant $\left(P\right.$ value $\left.=2.2 \times 10^{-16}\right)$. The agreement between the methods will increase with sample size because the Illumina $450 \mathrm{~K}$ methylation array has a technical resolution of approximately $17 \%$ for an individual sample. We also obtained lists of probes with thresholds of 20 and 25\% methylation difference. For both lists, the largest number of genes was again identified in the FFPE samples and the fewest in the PAXgene preserved specimens, along with a closer resemblance between PAXgene and fresh frozen tissues. The difference between probes identified in the FFPE or PAXgene and fresh frozen samples was again statistically significant $\left(P\right.$ value $\left.=2.2 \times 10^{-16}\right)$. The overall agreement can be divided into how sensitive and specific the preservation methods are. The two methods showed equal ability to identify the sites 


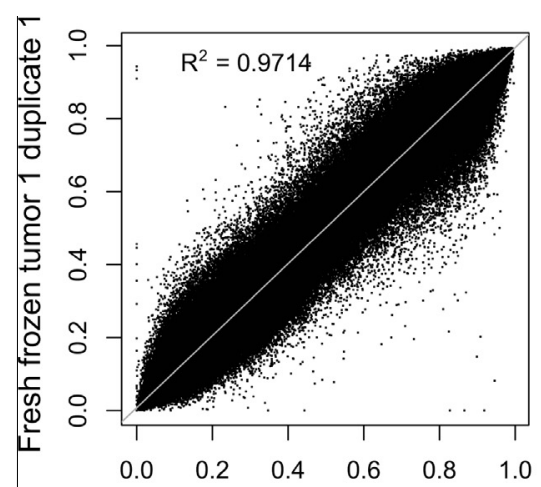

PAXgene tumor 1 duplicate 1

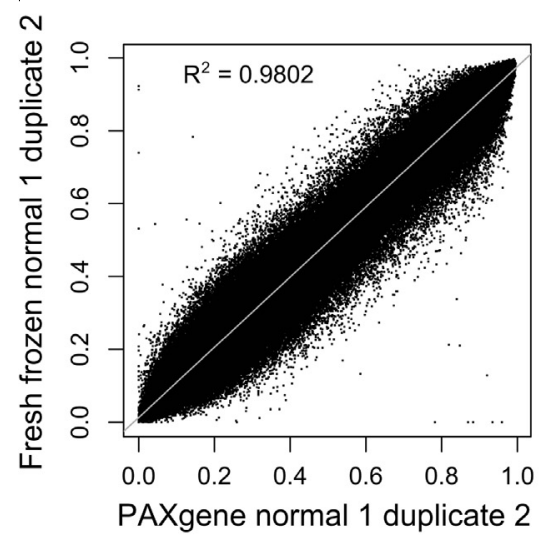

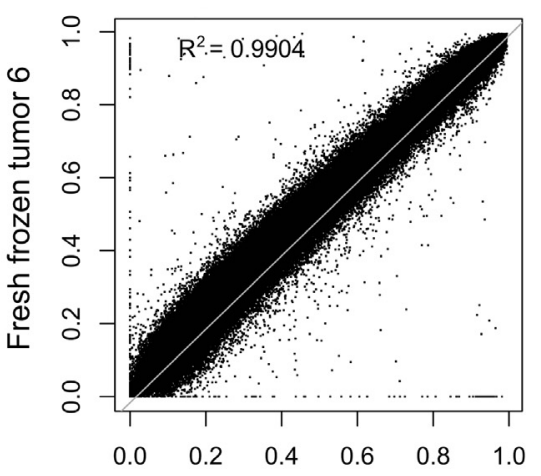

PAXgene tumor 6

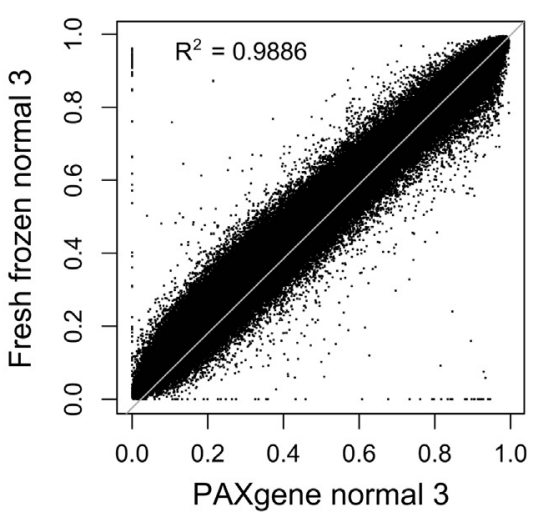

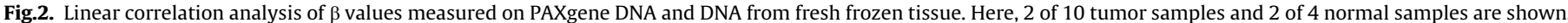
The coefficient of determination is displayed for each correlation analysis.
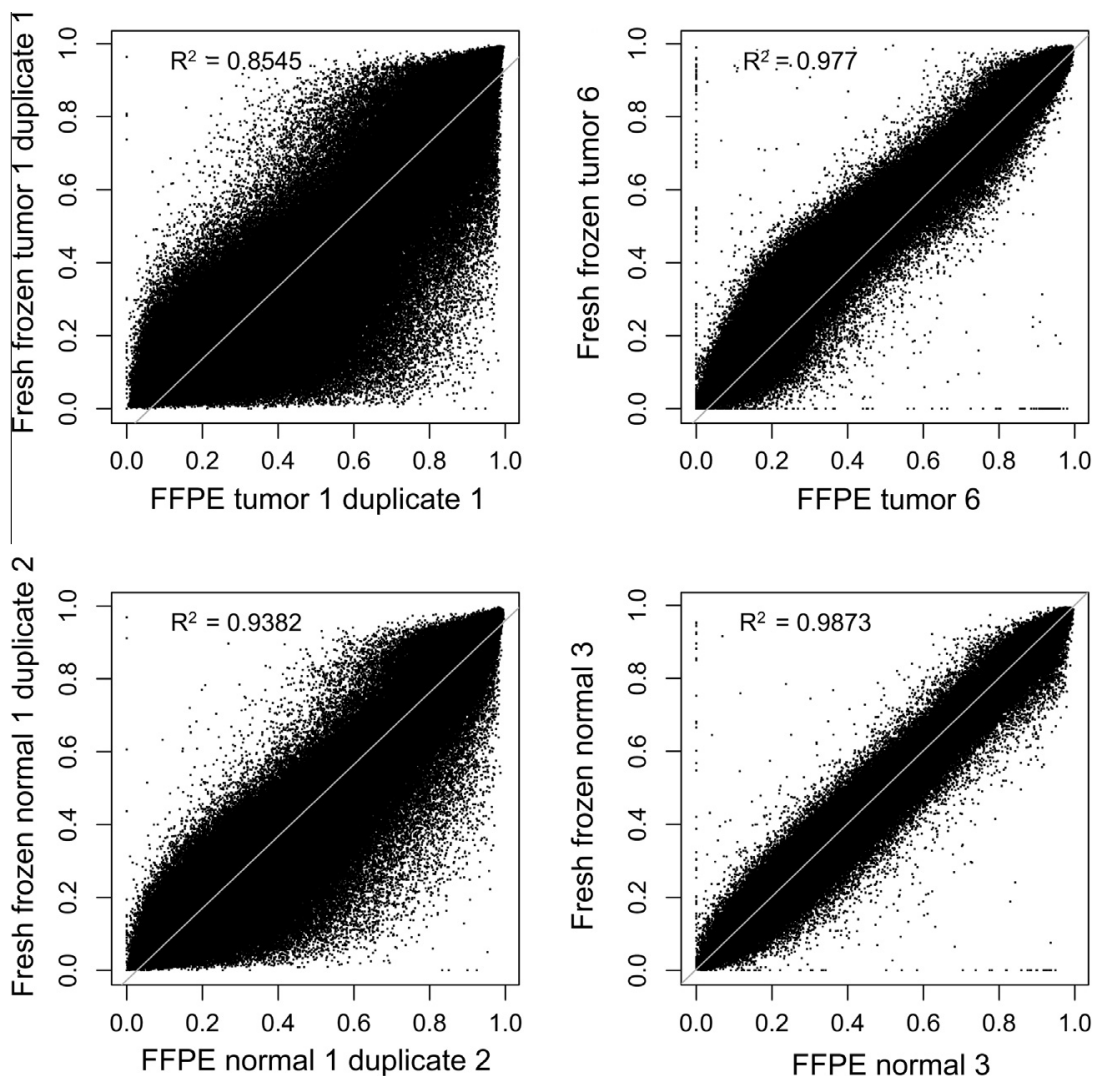

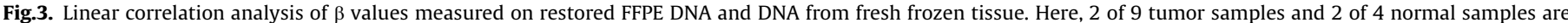
shown. The coefficient of determination is displayed for each correlation analysis. 
PAXgene Normal lung tissue

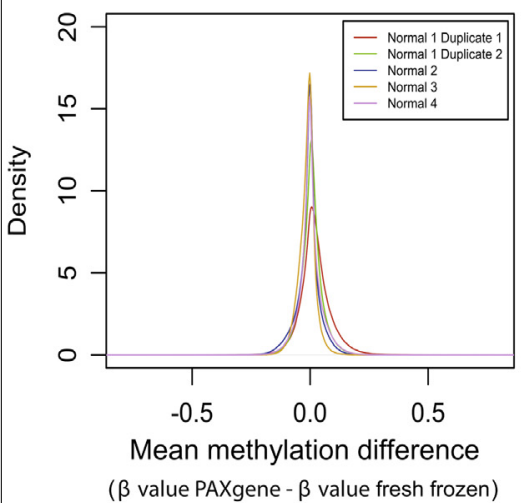

FFPE Normal lung tissue

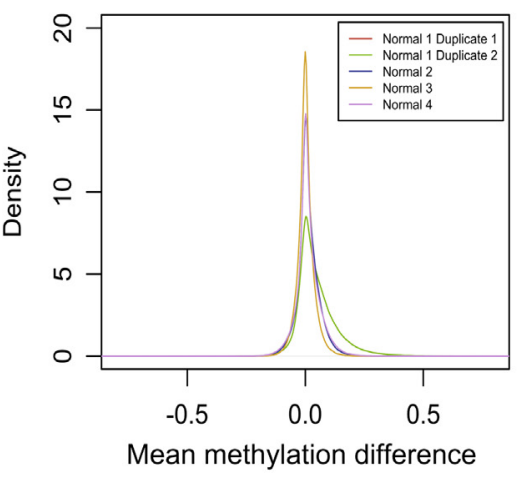

( $\beta$ value FFPE - $\beta$ value fresh frozen)
PAXgene Tumor lung tissue

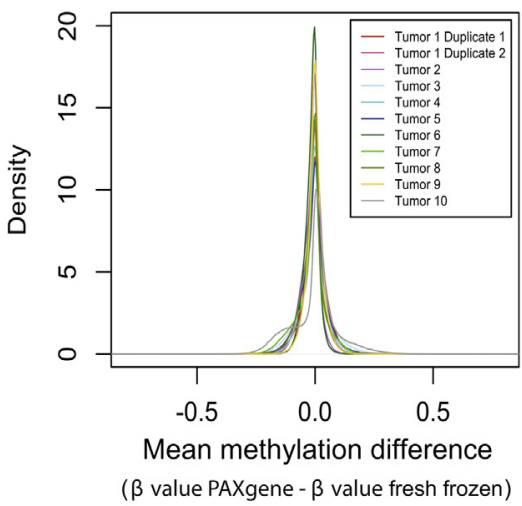

FFPE Tumor lung tissue

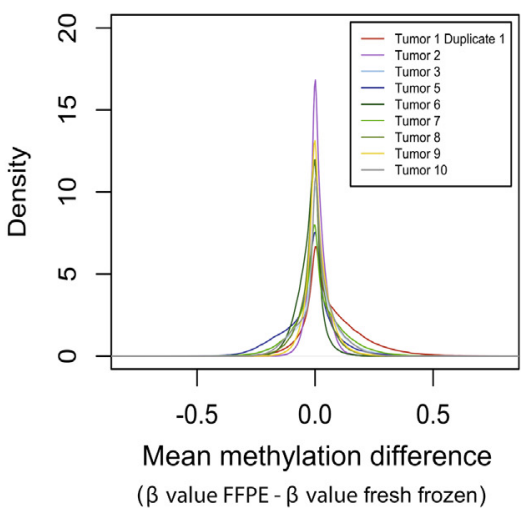

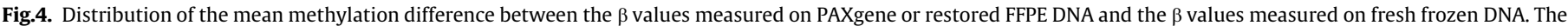
mean methylation differences for tumor and normal samples are displayed in separate graphs.

detected as differentially methylated in fresh frozen samples, giving a sensitivity of $39 \%$ for both PAXgene and FFPE. Conversely, the percentage of probes not observed in fresh frozen tissue was much higher for FFPE than for PAXgene, designating a strong overestimation of probes with altered DNA methylation patterns and, thereby, a much lower specificity in FFPE than in PAXgene preserved tissue ( 29 and $48 \%$, respectively). Furthermore, the location of the discordant probes was investigated to see whether any specific functional groups of probes were more prone to false positive or negative outcomes as a result of the preservation method. The probes incorrectly identified as differentially methylated (false positives), when using the results from the analysis in the fresh frozen samples as reference, had 346 of 761 (45\%) probes located in the CpG islands for FFPE preserved samples, whereas PAXgenefixed samples had 179 of 255 probes (70\%) in the same location. The probes missed as differentially methylated in either type of fixation (false negative results); FFPE had a large fraction located in the CpG islands (119 of 149 probes, $84 \%$ ), whereas PAXgene had 73 of 142 false negative probes (49\%) located in the CpG islands. No enrichment for other categories relative to the content of the array was observed.

In summary, the different analyses show convincingly that DNA isolated from PAXgene preserved tissues is proven to be superior to restored DNA from FFPE tissue but does not perfectly reflect the DNA methylation changes observed in the fresh frozen tissue.

\section{Discussion}

Despite its known inconvenience, formalin fixation is currently the standard for tissue preservation. Most pathology departments have collected large archives of FFPE tissue that represent an invaluable resource for future identification of biomarkers, especially in the light of the increasing focus on personalized medicine. Formalin fixation is cost-effective, preserves the morphology, and enables long-term storage of the samples. However, major drawbacks of formalin fixation are the fragmentation of nucleic acids and crosslinking of proteins and DNA, significantly reducing the quality of biomolecules $[12,13,29,30]$. The gold standard for quality preservation of nucleic acids and proteins is the snap-freezing method. However, this method is very time-consuming and the long-term storage is complicated given that the samples need to be stored at $-80^{\circ} \mathrm{C}$. Furthermore, snap-freezing compromises the morphology of the tissue. The non-crosslinking formalin-free PAXgene Tissue System was developed to improve the quality of nucleic acids without compromising the morphology of the tissue [13]. The preservation of DNA in PAXgene tissue is of similar molecular mass as DNA from snap-frozen tissue, and the RNA extracted from PAXgene tissue is also of high quality, with the same length of amplification products in PAXgene preserved and snap-frozen tissues $[10,12]$.

DNA from FFPE tissue performs suboptimally in genome-wide studies, such as the Infinium $450 \mathrm{~K}$ Methylation BeadChip, as a consequence of the highly degraded DNA. Restoration of the DNA to an acceptable quality is possible using either published protocols [23] or specialized kits, although these are cost-intensive and time-consuming. In this study, we have compared the performance of restored FFPE DNA on the Infinium 450K Methylation BeadChip with the performance of DNA from the PAXgene Tissue System, which can be used directly for the standard experimental protocol without prior restoration. We assessed the performance of the DNA 
Table 1

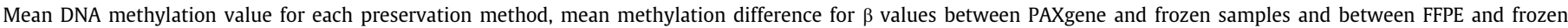
samples, and corresponding 95\% confidence intervals.

\begin{tabular}{|c|c|c|c|c|c|c|}
\hline Sample & Fixation method & Mean methylation & $\triangle$ PAXgene & $\triangle$ PAXgene $95 \% C I$ & $\triangle \mathrm{FFPE}$ & $\triangle$ FFPE $95 \% C I$ \\
\hline Tumor 1 & PAXgene & 0.4835 & -0.0014 & $(-0.0016,-0.0013)$ & 0.0675 & $(0.0671,0.0678)$ \\
\hline \multirow[t]{2}{*}{ Duplicate 1} & Fresh frozen & 0.4850 & & & & \\
\hline & FFPE & 0.5524 & & & & \\
\hline Tumor 1 & PAXgene & 0.5013 & -0.0008 & $(-0.0009,-0.0007)$ & 0.0666 & $(0.0658,0.0674)$ \\
\hline \multirow[t]{2}{*}{ Duplicate 2} & Fresh frozen & 0.5020 & & & & \\
\hline & FFPE & 0.5687 & & & & \\
\hline \multirow[t]{3}{*}{ Tumor 2} & PAXgene & 0.5466 & -0.0173 & $(-0.0174,-0.0172)$ & 0.0089 & $(0.0089,0.0091)$ \\
\hline & Fresh frozen & 0.5640 & & & & \\
\hline & FFPE & 0.5729 & & & & \\
\hline \multirow[t]{3}{*}{ Tumor 3} & PAXgene & 0.5146 & -0.0041 & $(-0.0043,-0.0039)$ & 0.0083 & $(0.0080,0.0086)$ \\
\hline & Fresh frozen & 0.5187 & & & & \\
\hline & FFPE & 0.5270 & & & & \\
\hline \multirow[t]{2}{*}{ Tumor 4} & PAXgene & 0.5099 & -0.0062 & $(-0.0064,-0.0061)$ & - & - \\
\hline & Fresh frozen & 0.5161 & & & & \\
\hline \multirow[t]{3}{*}{ Tumor 5} & PAXgene & 0.5374 & -0.0062 & $(-0.0064,-0.0061)$ & -0.0227 & $(-0.0230,-0.0224)$ \\
\hline & Fresh frozen & 0.5437 & & & & \\
\hline & FFPE & 0.5209 & & & & \\
\hline \multirow[t]{3}{*}{ Tumor 6} & PAXgene & 0.4708 & -0.0137 & $(-0.0138,-0.0136)$ & -0.0225 & $(-0.0227,-0.0224)$ \\
\hline & Fresh frozen & 0.4845 & & & & \\
\hline & FFPE & 0.4620 & & & & \\
\hline \multirow[t]{3}{*}{ Tumor 7} & PAXgene & 0.5143 & -0.0159 & $(-0.0161,-0.0157)$ & 0.0105 & $(0.0103,0.0108)$ \\
\hline & Fresh frozen & 0.5302 & & & & \\
\hline & FFPE & 0.5408 & & & & \\
\hline \multirow[t]{3}{*}{ Tumor 8} & PAXgene & 0.5506 & -0.0141 & $(-0.0143,-0.0140)$ & -0.004 & $(-0.0042,-0.0039)$ \\
\hline & Fresh frozen & 0.5647 & & & & \\
\hline & FFPE & 0.5607 & & & & \\
\hline \multirow[t]{3}{*}{ Tumor 9} & PAXgene & 0.5202 & -0.0012 & $(-0.0013,-0.0010)$ & 0.0106 & $(0.0104,0.0107)$ \\
\hline & Fresh frozen & 0.5213 & & & & \\
\hline & FFPE & 0.5320 & & & & \\
\hline \multirow[t]{3}{*}{ Tumor 10} & PAXgene & 0.5208 & -0.0017 & $(-0.0020,-0.0014)$ & 0.0093 & $(0.0091,0.0095)$ \\
\hline & Fresh frozen & 0.5225 & & & & \\
\hline & FFPE & 0.5318 & & & & \\
\hline Normal 1 & PAXgene & 0.4996 & 0.02134 & $(0.0212,0.0215)$ & 0.0473 & $(0.0471,0.0476)$ \\
\hline \multirow[t]{2}{*}{ Duplicate 1} & Fresh frozen & 0.4783 & & & & \\
\hline & FFPE & 0.5256 & & & & \\
\hline Normal 1 & PAXgene & 0.4819 & 0.047 & $(0.0046,0.0049)$ & 0.0324 & $(0.0322,0.0327)$ \\
\hline \multirow[t]{2}{*}{ Duplicate 2} & Fresh frozen & 0.4772 & & & & \\
\hline & FFPE & 0.5107 & & & & \\
\hline \multirow[t]{3}{*}{ Normal 2} & PAXgene & 0.4955 & -0.0076 & $(-0.0078,-0.0075)$ & 0.012 & $(0.0119,0.0121)$ \\
\hline & Fresh frozen & 0.5031 & & & & \\
\hline & FFPE & 0.5151 & & & & \\
\hline \multirow[t]{3}{*}{ Normal 3} & PAXgene & 0.4928 & -0.0129 & $(-0.0130,-0.0128)$ & 0.0019 & $(0.0018,0.0020)$ \\
\hline & Fresh frozen & 0.5057 & & & & \\
\hline & FFPE & 0.5076 & & & & \\
\hline \multirow[t]{3}{*}{ Normal 4} & PAXgene & 0.5065 & 0.0008 & $(0.0007,0.0010)$ & 0.0118 & $(0.0117,0.0120)$ \\
\hline & Fresh frozen & 0.5067 & & & & \\
\hline & FFPE & 0.5175 & & & & \\
\hline
\end{tabular}

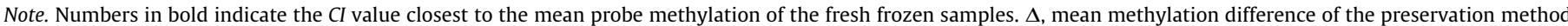
compared with the corresponding fresh frozen sample; $C I$, confidence interval.

methylation analysis in FFPE, PAXgene, and frozen tissue samples from the same clinical specimen.

To our knowledge, this is the first demonstration of DNA methylation analysis in PAXgene-fixed tissues. The superior quality of PAXgene DNA was demonstrated by the determination of the limit of agreement between the methods. Differences between PAXgene and frozen samples were lower in 8 of 13 samples compared with FFPE samples, and only 1 FFPE sample had a closer resemblance to the DNA methylation detected in the fresh frozen sample compared with PAXgene preserved DNA. The mean methylation difference also showed a difference closer to zero between PAXgene and frozen samples and smaller 95\% CI values compared with FFPE samples. Thus, in summary, our results do confirm the high DNA quality obtained when using PAXgene fixation, although it should not be neglected that, despite the close resemblance, the PAXgene DNA methylation measures were not exactly the same as the DNA methylation measures in the fresh frozen samples.

Although we convincingly showed in our study using multiple analyses that PAXgene fixation shows improved results compared with restored DNA from FFPE samples, our data are not in disagreement with two recent publications evaluating the use of restored DNA from FFPE samples that came to the conclusion that restored FFPE DNA is suited for 450K-based DNA methylation analysis [31,32]. Although the data from Moran and coworkers were normalized, which should reduce the variability between FFPE and frozen samples, the resemblance of samples from different fixation methods originating from the same tumor is clearly visible in the hierarchical clustering in their study [32] as well as in our study. On the other hand, the study by Dumenil and coworkers pointed out that, despite a normalization procedure, the restored FFPE samples are different from the frozen samples, and although the differential DNA methylation data point to the same biological pathways, studies must be performed on either frozen or restored FFPE samples and it is currently not advisable to mix restored FFPE and fresh frozen samples in a single study [31]. The results of our study are in full agreement with this statement.

It should be noted that neither of these two studies calculated the limit of agreement between fresh frozen and restored DNA 
Table 2

Limit of agreement intervals for difference of $\beta$ values between PAXgene or FFPE fixed samples and frozen samples.

\begin{tabular}{|c|c|c|c|c|}
\hline Sample & $\begin{array}{l}\text { PAXgene lower limit of agreement } \\
\text { (CI for lower limit) }\end{array}$ & $\begin{array}{l}\text { PAXgene higher limit of agreement } \\
\text { (CI for higher limit) }\end{array}$ & $\begin{array}{l}\text { FFPE lower limit of agreement } \\
\text { (CI for lower limit) }\end{array}$ & $\begin{array}{l}\text { FFPE higher limit of agreement } \\
\text { ( } C I \text { for higher limit) }\end{array}$ \\
\hline Tumor 1 & -0.1111 & 0.1082 & -0.1791 & 0.3141 \\
\hline Duplicate 1 & $(-0.1114,-0.1108)$ & $(0.1079,0.1085)$ & $(-0.1797,-0.1785)$ & $(0.3135,0.3147)$ \\
\hline Tumor 1 & -0.0792 & 0.0777 & -0.5056 & 0.6388 \\
\hline Duplicate 2 & $(-0.0794,-0.0791)$ & $(0.0775,0.0779)$ & $(-0.5070,-0.5042)$ & $(0.6374,0.6402)$ \\
\hline Tumor 2 & $\begin{array}{l}-0.0924 \\
(-0.0926,-0.0922)\end{array}$ & $\begin{array}{l}0.0577 \\
(0.0576,0.0579)\end{array}$ & $\begin{array}{l}-0.0705 \\
(-0.0707,-0.0703)\end{array}$ & $\begin{array}{l}0.0885 \\
(0.0883,0.0887)\end{array}$ \\
\hline Tumor 3 & $\begin{array}{l}-\mathbf{0 . 1 4 2 8} \\
(-0.1431,-0.1425)\end{array}$ & $\begin{array}{l}\mathbf{0 . 1 3 4 6} \\
(0.1343,0.1349)\end{array}$ & $\begin{array}{l}-0.1721 \\
(-0.1725,-0.1716)\end{array}$ & $\begin{array}{l}0.1887 \\
(0.1882,0.1891)\end{array}$ \\
\hline Tumor 4 & $\begin{array}{l}-0.1113 \\
(-0.1115,-0.1111)\end{array}$ & $\begin{array}{l}0.0989 \\
(0.0987,0.0992)\end{array}$ & - & - \\
\hline Tumor 5 & $\begin{array}{l}-\mathbf{0 . 1 2 5 5} \\
(-0.1258,-0.1252)\end{array}$ & $\begin{array}{l}\mathbf{0 . 1 1 3 0} \\
(0.1127,0.1133)\end{array}$ & $\begin{array}{l}-0.2392 \\
(-0.2398,-0.2387)\end{array}$ & $\begin{array}{l}0.1938 \\
(0.1933,0.1943)\end{array}$ \\
\hline Tumor 6 & $\begin{array}{l}-\mathbf{0 . 0 8 0 1} \\
(-0.0803,-0.0800)\end{array}$ & $\begin{array}{l}\mathbf{0 . 0 5 2 7} \\
(0.0525,0.0529)\end{array}$ & $\begin{array}{l}-0.1275 \\
(-0.1277,-0.1272)\end{array}$ & $\begin{array}{l}0.0824 \\
(0.0822,0.0827)\end{array}$ \\
\hline Tumor 7 & $\begin{array}{l}-\mathbf{0 . 1 3 8 6} \\
(-0.1389,-0.1383)\end{array}$ & $\begin{array}{l}\mathbf{0 . 1 0 6 8} \\
(0.1065,0.1071)\end{array}$ & $\begin{array}{l}-0.1966 \\
(-0.1972,-0.1961)\end{array}$ & $\begin{array}{l}0.2177 \\
(0.2172,0.2182)\end{array}$ \\
\hline Tumor 8 & $\begin{array}{l}-\mathbf{0 . 1 1 1 1} \\
(-0.1113,-0.1108)\end{array}$ & $\begin{array}{l}\mathbf{0 . 0 8 2 8} \\
(0.0826,0.0830)\end{array}$ & $\begin{array}{l}-0.1318 \\
(-0.1321,-0.1315)\end{array}$ & $\begin{array}{l}0.1237 \\
(0.1234,0.1240)\end{array}$ \\
\hline Tumor 9 & $\begin{array}{l}-\mathbf{0 . 0 7 8 7} \\
(-0.0789,-0.0785)\end{array}$ & $\begin{array}{l}\mathbf{0 . 0 7 6 4} \\
(0.0762,0.0766)\end{array}$ & $\begin{array}{l}-0.1005 \\
(-0.1008,-0.1002)\end{array}$ & $\begin{array}{l}0.1216 \\
(0.1214,0.1219)\end{array}$ \\
\hline Tumor 10 & $\begin{array}{l}-0.1925 \\
(-0.1929,-0.1920)\end{array}$ & $\begin{array}{l}0.1891 \\
(0.1886,0.1896)\end{array}$ & $\begin{array}{l}-\mathbf{0 . 1 4 7 3} \\
(-0.1477,-0.1469)\end{array}$ & $\begin{array}{l}\mathbf{0 . 1 6 5 9} \\
(0.1655,0.1663)\end{array}$ \\
\hline Normal 1 & -0.1097 & 0.1524 & -0.1143 & 0.2090 \\
\hline Duplicate 1 & $(-0.1100,-0.1094)$ & $(0.1521,0.1527)$ & $(-0.1147,-0.1139)$ & $(0.2086,0.2094)$ \\
\hline Normal 1 & -0.0936 & 0.1030 & -0.1344 & 0.2013 \\
\hline Duplicate 2 & $(-0.0938,-0.0933)$ & $(0.1028,0.1032)$ & $(-0.1348,-0.1340)$ & $(0.2010,0.2018)$ \\
\hline Normal 2 & $\begin{array}{l}-0.1061 \\
(-0.1064,-0.1059)\end{array}$ & $\begin{array}{l}0.0908 \\
(0.0906,0.0911)\end{array}$ & $\begin{array}{l}-0.0828 \\
(-0.0831,-0.0826)\end{array}$ & $\begin{array}{l}0.1069 \\
(0.1066,0.1071)\end{array}$ \\
\hline Normal 3 & $\begin{array}{l}-0.0878 \\
(-0.0880,-0.0876)\end{array}$ & $\begin{array}{l}0.0620 \\
(0.0618,0.0622)\end{array}$ & $\begin{array}{l}-0.0738 \\
(-0.0740,-0.0736)\end{array}$ & $\begin{array}{l}0.0776 \\
(0.0774,0.0778)\end{array}$ \\
\hline Normal 4 & $\begin{array}{l}-0.0995 \\
(-0.0998,-0.0992)\end{array}$ & $\begin{array}{l}0.1012 \\
(0.1009,0.1014)\end{array}$ & $\begin{array}{l}-0.0907 \\
(-0.0910,-0.0905)\end{array}$ & $\begin{array}{l}0.1143 \\
(0.1141,0.1146)\end{array}$ \\
\hline
\end{tabular}

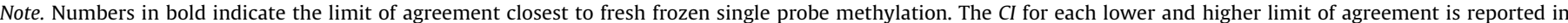
parentheses. CI, confidence interval.

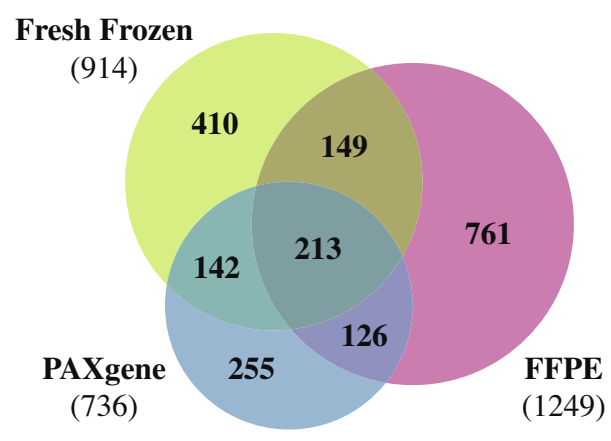

Fig.5. Venn diagram of the probes detected with more than $30 \%$ methylation difference and a $P$ value less than 0.05 between lung tumors and normal lung tissue in fresh frozen, PAXgene, and FFPE tissues.

from FFPE samples, which would have allowed an unbiased assessment of the performance and comparative analysis of the three studies, and that analysis was restricted to a subset of the probes present on the array, in contrast to our study.

Even though PAXgene preserved DNA, RNA, and proteins are of considerable higher quality compared with those purified from FFPE tissue, the change from formalin to PAXgene fixation in the daily clinic would require a major restructuring. In addition, it should not be neglected that PAXgene fixation has some drawbacks compared with FFPE fixation. First, the consumables are more expensive compared with formalin fixation. However, when fixating small samples, only 2 to $4 \mathrm{~h}$ of fixation is needed for PAXgene treatment compared with 24 to $48 \mathrm{~h}$ for formalin, improving the efficiency in the laboratory. Second, fixation is limited to small sample sizes with a limit of $4 \times 10 \times 10 \mathrm{~mm}$. Third, and most important, PAXgene blocks need to be stored at $4{ }^{\circ} \mathrm{C}$ [12]. In addition, long-term storage (e.g., 15 years) still needs to be assessed for PAXgene fixation to see how this affects both the biomolecules and the morphology. Nonetheless, it might prove valuable, especially if biological samples are collected with the aim of comprehensive analyses as, for example, in clinical trials.

For the genome-wide analysis of DNA methylation patterns using the Infinium $450 \mathrm{~K}$ array, we showed in this study that DNA from PAXgene preserved clinical specimens performs considerably better compared with restored FFPE DNA. Furthermore, FFPE DNA needs to be restored prior to the array analysis. This restoration is time-consuming and expensive, and it largely outweighs the extra expenses associated with PAXgene fixation, which can be directly used on the array. Furthermore, despite an initial quality control step, 1 sample failed restoration and was not usable for downstream analysis. The high performance of the DNA from PAXgene-fixed tissue on the Infinium 450K Methylation BeadChip also suggests that the PAXgene preserved DNA may also be directly used on many other array-based hybridization analyses, for which FFPE DNA performs inadequately.

\section{Conclusions}

We have demonstrated the excellent quality of DNA from PAXgene preserved tissue for DNA methylation analysis and that, for genome-wide DNA methylation analysis, it performs better compared with restored FFPE DNA. In addition, DNA from PAXgene can be directly used on the array without prior restoration, rendering the analytical process significantly more time- and cost-effective. Our results also suggest that PAXgene preserved DNA may also be suitable for other genome-wide analyses. 


\section{Acknowledgments}

The research leading to the described results received funding from the A.P. Møller and Chastine McKinney Møller Foundation and the King Christian X Foundation. The authors thank Celine Besse and Marie-Therese Bihoreau (Centre National de Génotypage) for performing the epigenotyping experiments.

\section{Appendix A. Supplementary data}

Supplementary data associated with this article can be found, in the online version, at http://dx.doi.org/10.1016/j.ab.2014.09.012.

\section{References}

[1] G. Poste, Bring on the biomarkers, Nature 469 (2011) 156-157.

[2] C.H. Fox, F.B. Johnson, J. Whiting, P.P. Roller, Formaldehyde fixation, J. Histochem. Cytochem. 33 (1985) 845-853.

[3] J.R. Kuykendall, M.S. Bogdanffy, Efficiency of DNA-histone crosslinking induced by saturated and unsaturated aldehydes in vitro, Mutat. Res. 283 (1992) 131-136.

[4] C. Williams, F. Pontén, C. Moberg, P. Söderkvist, M. Uhlen, J. Ponten, G. Sitbon, J. Lundeberg, A high frequency of sequence alterations is due to formalin fixation of archival specimens, Am. J. Pathol. 155 (1999) 1467-1471.

[5] D. von Smolinski, I. Leverkoehne, G. von Samson-Himmelstjerna, A.D. Gruber, Impact of formalin-fixation and paraffin-embedding on the ratio between mRNA copy numbers of differently expressed genes, Histochem. Cell Biol. 124 (2005) 177-188.

[6] D.L. Evers, C.B. Fowler, B.R. Cunningham, J.T. Mason, T.J. O’Leary, The effect of formaldehyde fixation on RNA: optimization of formaldehyde adduct removal, J. Mol. Diagn. 13 (2011) 282-288.

[7] B. Metz, G.F. Kersten, G.J. Baart, A. de Jong, H. Meiring, J. ten Hove, M.J. van Steenbergen, W.E. Hennink, D.J. Crommelin, W. Jiskoot, Identification of formaldehyde-induced modifications in proteins: reactions with model peptides, J. Biol. Chem. 279 (2004) 6235-6243.

[8] E.B. Desciak, M.E. Maloney, Artifacts in frozen section preparation, Dermatol. Surg. 26 (2000) 500-504.

[9] S.R. Shi, C. Liu, L. Pootrakul, L. Tang, A. Young, R. Chen, R.J. Cote, C.R. Taylor, Evaluation of the value of frozen tissue section used as "gold standard" for immunohistochemistry, Am. J. Clin. Pathol. 129 (2008) 358-366.

[10] C. Viertler, D. Groelz, S. Gündisch, K. Kashofer, B. Reischauer, P.H. Riegman, R. Winther, R. Wyrich, K.F. Becker, U. Oelmüller, et al., A new technology for stabilization of biomolecules in tissues for combined histological and molecular analyses, J. Mol. Diagn. 14 (2012) 458-466.

[11] M. Kap, F. Smedts, W. Oosterhuis, R. Winther, N. Christensen, B. Reischauer, C. Viertler, D. Groelz, K.-F. Becker, K. Zatloukal, et al., Histological assessment of PAXgene tissue fixation and stabilization reagents, PLoS One 6 (2011) e27704.

[12] B. Belloni, C. Lambertini, P. Nuciforo, J. Phillips, E. Bruening, S. Wong, R. Dummer, Will PAXgene substitute formalin? A morphological and molecular comparative study using a new fixative system, J. Clin. Pathol. 66 (2013) 124135.
[13] B. Ergin, S. Meding, R. Langer, M. Kap, C. Viertler, C. Schott, U. Ferch, P. Riegman, K. Zatloukal, A. Walch, et al., Proteomic analysis of PAXgene-fixed tissues, J. Proteome Res. 9 (2010) 5188-5196.

[14] D. Hanahan, R.A. Weinberg, Hallmarks of cancer: the next generation, Cell 144 (2011) 646-674.

[15] M.A. Dawson, T. Kouzarides, Cancer epigenetics: from mechanism to therapy, Cell 150 (2012) 12-27.

[16] P.T. Tan, A.H. Wei, The epigenomics revolution in myelodysplasia: a clinicopathological perspective, Pathology 43 (2011) 536-546.

[17] Y. Kanai, Alterations of DNA methylation and clinico-pathological diversity of human cancers, Pathol. Int. 58 (2008) 544-558.

[18] X. Deng, F. Campagne, Introduction to the development and validation of predictive biomarker models from high-throughput data sets, Methods Mol. Biol. 620 (2010) 435-470.

[19] A. How Kit, H.M. Nielsen, J. Tost, DNA methylation based biomarkers: practical considerations and applications, Biochimie 94 (2012) 2314-2337.

[20] G.H. Kim, J.J. Ryan, S.L. Archer, The role of redox signaling in epigenetics and cardiovascular disease, Antioxid. Redox Signal. 18 (2013) 1920-1936.

[21] L. Chouliaras, D. Mastroeni, E. Delvaux, A. Grover, G. Kenis, P.R. Hof, H.W. Steinbusch, P.D. Coleman, B.P. Rutten, D.L. van den Hove, Consistent decrease in global DNA methylation and hydroxymethylation in the hippocampus of Alzheimer's disease patients, Neurobiol. Aging 34 (2013) 2091-2099.

[22] J. Sandoval, H. Heyn, S. Moran, J. Serra-Musach, M.A. Pujana, M. Bibikova, M. Esteller, Validation of a DNA methylation microarray for 450,000 CpG sites in the human genome, Epigenetics 6 (2011) 692-702.

[23] C. Thirlwell, M. Eymard, A. Feber, A. Teschendorff, K. Pearce, M. Lechner M. Widschwendter, S. Beck, Genome-wide DNA methylation analysis of archival formalin-fixed paraffin-embedded tissue using the Illumina Infinium HumanMethylation27 BeadChip, Methods 52 (2010) 248-254.

[24] F. Jasmine, R. Rahaman, S. Roy, M. Raza, R. Paul, M. Rakibuz-Zaman, R. PaulBrutus, C. Dodsworth, M. Kamal, H. Ahsan, et al., Interpretation of genomewide infinium methylation data from ligated DNA in formalin-fixed, paraffinembedded paired tumor and normal tissue, BMC Res. Notes 5 (2012) 117.

[25] StataCorp, Stata Statistical Software: Release 13, StataCorp, College Station, TX 2013.

[26] R Core Team, R: a language and environment for statistical computing, $\mathrm{r}$ foundation for statistical computing, Vienna, Austria, <http://www.Rproject.org>, 2013.

[27] B. Carstensen, Comparing Clinical Measurement Methods: A Practical Guide, vol. 1, John Wiley, New York, 2010. p. 172.

[28] J.M. Bland, D.G. Altman, Statistical methods for assessing agreement between two methods of clinical measurement, Lancet 1 (1986) 307-310.

[29] G. Stanta, S.P. Mucelli, F. Petrera, S. Bonin, G. Bussolati, A novel fixative improves opportunities of nucleic acids and proteomic analysis in human archive's tissues, Diagn. Mol. Pathol. 15 (2006) 115-123.

[30] H. Puchtler, S.N. Meloan, On the chemistry of formaldehyde fixation and its effects on immunohistochemical reactions, Histochemistry 82 (1985) 201 204.

[31] T.D. Dumenil, L.F. Wockner, M. Bettington, D.M. McKeone, K. Klein, L.M Bowdler, G.W. Montgomery, B.A. Leggett, V.L. Whitehall, Genome-wide DNA methylation analysis of formalin-fixed paraffin embedded colorectal cancer tissue, Genes Chromosom. Cancer 53 (2014) 537-548.

[32] S. Moran, M. Vizoso, A. Martinez-Cardús, A. Gomez, X. Matías-Guiu, S.M. Chiavenna, A.G. Fernandez, M. Esteller, Validation of DNA methylation profiling in formalin-fixed paraffin-embedded samples using the Infinium HumanMethylation450 microarray, Epigenetics 9 (2014) 829-833. 